\title{
A PALEY-WIENER THEOREM FOR LOCALLY COMPACT ABELIAN GROUPS
}

\author{
BY
}

GUNAR E. LIEPINS

\begin{abstract}
Extending the Paley-Wiener theorem to locally compact Abelian groups involves both finding a suitable Laplace transform and a suitable analogue for analytic functions. The Laplace transform is defined in terms of complex characters, and differentiability is defined with use of one-parameter subgroups. The resulting theorem is much as conjectured by Mackey [7], $\left.{ }^{1}\right)$ the major differences being that the theorem is very much an $L^{2}$ theorem and that the problem exhibits a surprising finite dimensional nature.
\end{abstract}

\section{Definitions.}

Definition 1. Let $G$ be a locally compact Abelian group. Then a complex character $z$ on $G$ is a continuous, nonzero, complex valued homomorphism.

It is clear that every complex character $z$ has a polar decomposition $z=x y$, where $x$ is a continuous positive valued homorphism, henceforth called a real character, and $y$ is a unitary character in the sense of Pontrjagin [10].

Conversely, it is clear that a real character $x$ and a unitary character $y$ determine a complex character by $z=x y$.

Definition 2. Let $G$ be a locally compact Abelian group. A continuous homomorphism $r$ from $G$ into the additive reals $R$ is called a linear functional. The set of linear functionals on $G$ will be called $G^{\#}$, and the topology of $G^{\#}$ will be understood to be the compact-open topology induced by $G$.

It is obvious that for any real character $x, \log x$ is a linear functional, and conversely, for any linear functional $r, \exp r$ is a real character. Thus there exists a bijection between the real characters and the linear functionals, and by abuse of notation, we shall call both the real character $x$ and its associated linear functional by the symbol $x$.

Presented to the Society, January 23, 1975 under the title A Paley-Wiener theorem; received by the editors May 2, 1975.

AMS (MOS) subject classifications (1970). Primary 43A32, 44A10. character.

Key words and phrases. Locally compact Abelian groups, Laplace transform, group

(1) This paper is essentially the author's doctoral dissertation at Dartmouth College and was supported in part by a Dartmouth College Fellowship and a research grant from the office of the Vice President for Research and Graduate Studies at Texas Tech University. The author wishes to express his warm thanks to his thesis advisor, Professor Reese T. Prosser. 
To every $x \in G^{\#}$, and every $u \in R$, we can associate a function $\psi_{x}(u)(\cdot)$ taking an element $t \in G$ into the complex number $\exp (\operatorname{iux}(t))$. It is immediate that $\psi_{x}(u)(\cdot)$ determines a unitary character and that the map $\psi_{x}: R \rightarrow G^{*}$ defined by $\psi_{x}(u)=\psi_{x}(u)(\cdot)$ is a one-parameter subgroup of the character group $G^{*}$ (where $G^{*}$ has the compact-open topology).

LEMMA $1\left[11\right.$, p. 12]. Let $G$ be a locally compact Abelian group and $G^{\#}$ the space of linear functionals on $G$, and $G^{*}$ the character group of $G$. Then $G^{\#}$ can be identified with the one-parameter subgroups of $G^{*}$.

If $x$ is a linear functional, $t \in G$ and $u$ is a real number, then $x(t)$ will denote the value at $t$ of $x$, and $x[u]$ will denote the element of $G^{*}$ assigned to $u$ by the one-parameter subgroup associated with $x$. Noting that $\left(x_{1}+x_{2}\right)[u]=$ $x_{1}[u] x_{2}[u]$ and $\left(u_{1} x\right)[u]=x\left[u_{1} u\right]$, where $x, x_{1}, x_{2}$ are linear functionals and $u, u_{1}$ are real numbers, we can conclude that the set of linear functionals can be considered to be a real vector space. The question about the existence of functionals is answered by the following

LEMMA 2 [7, p. 157], [4, pp. 378-380]. Let $G$ be a locally compact Abelian group, $G^{*}$ its character group. Then the following statements about $G$ and $G^{*}$ are equivalent.

(i) For each $t$ in $G$ distinct from 0 , there exists a real character $x$ with $x(t) \neq 1$.

(ii) For each $t$ in $G$ distinct from 0 , there exists a functional $x$ with $x(t)$ $\neq 0$.

(iii) The union of the images of all one-parameter subgroups of $G^{*}$ is dense in $G^{*}$.

(iv) $G^{*}$ is connected.

(v) $G$ is the direct product of a discrete torsion free group $D$ and the additive group of an $n$-dimensional vector space $V$.

COROLlaRY 2.1. Let $G$ be a locally compact Abelian group, and $N$ the subgroup of $G$ on which all $x \in G^{\#}$ vanish identically; then $G / N$ is isomorphic to $D \oplus V$ (notation of Lemma 2).

To facilitate later calculations certain additional facts about the space $G^{\#}$ are useful.

Lemma 3. Let $G$ be a locally compact Abelian group and $N$ as in Corollary 2.1. Then $G^{\#}$ can be imbedded in $(G / N)^{\#}$.

Proof. Let $i$ be the natural quotient map, $i: G \rightarrow G / N$. For $t \in G$, the equality $\mathrm{x}(i(t))=x(t)$ defines an isomorphism between $\mathrm{x} \in(G / N)^{\#}$ and $x \in G^{\#}$ $[8$, p. 27]. 
LEMMA 4. Let $G_{1}, G_{2}$ be two locally compact Abelian groups with linear functional spaces $G_{1}^{\#}, G_{2}^{\#}$ respectively. Then $G_{1}^{\#} \times G_{2}^{\#}$ is isomorphic with $\left(G_{1} \oplus G_{2}\right)^{\#}$.

Proof. Let $x_{i} \in G_{i}^{\#}$. Then the map $\theta$ taking the pair $\left(x_{1}, x_{2}\right)$ to $x_{1} \oplus$ $x_{2} \in\left(G_{1} \oplus G_{2}\right)^{\#}$, defined by $x_{1} \oplus x_{2}\left(t_{1} \oplus t_{2}\right)=x_{1}\left(t_{1}\right)+x_{2}\left(t_{2}\right)$, is a vector space isomorphism of $G_{1}^{\#} \times G_{2}^{\#}$ with its image in $\left(G_{1} \oplus G_{2}\right)^{\#}$. Now let $\mathbf{x}$ be any element of $\left(G_{1} \oplus G_{2}\right)^{\#}$. Define $x_{1} \in G_{1}^{\#}$ by $x_{1}(t)=\mathrm{x}(t, 0)$ and $x_{2} \in G_{2}^{\#}$ by $x_{2}(t)=\mathrm{x}(0, t)$. Hence $\theta$ is an isomorphism onto $\left(G_{1} \oplus G_{2}\right)^{\#}$.

Laplace transforms. The $L^{2}$ Laplace transform of a suitably restricted function $f$ is defined by replacing the unitary characters of the Fourier transform by the complex characters previously described. In view of our earlier remarks we write

$$
f^{\square}(x, y)=\int_{G} f(t) \exp (x(t))\langle y, t\rangle d \mu(t)
$$

where $y \in G^{*}, x \in G^{\#}$ and $d \mu$ is the Haar measure, and the integral is defined at least for $f \in C_{c}(G)$ (continuous functions with compact support).

LemMA 5. Let $G$ be a locally compact Abelian group and $f$ be any square integrable function on $G\left(f \in L^{2}(G)\right)$. The set $U_{f}$ of all $x \in G^{\#}$ for which $f \exp x$ is square integrable is a convex subset of $G^{\#}$ which contains the zero element.

Proof. Let $x_{1}, x_{2} \in U_{f}$. Let $G_{1}=\left\{t \in G: x_{1}(t) \geqslant x_{2}(t)\right\}, G_{2}=G \sim G_{1}$. Let $x_{3}=\alpha x_{1}+(1-\alpha) x_{2}$ for $0 \leqslant \alpha \leqslant 1$. On $G_{i}$,

$$
\left|f(t) \exp \left(x_{3}(t)\right)\right|^{2} \leqslant\left|f(t) \exp \left(x_{i}(t)\right)\right|^{2},
$$

and hence $x_{3} \in U_{f}$.

Definition 3. A function $f \in L^{2}(G)$ for which $U_{f}$ contains a neighborhood $U$ of 0 in $G^{\#}$ is defined to be strongly $L^{2}$ and is denoted $f \in S L^{2}(G)$.

REMARKS. (i) $S L^{2}(G)$ is a linear space.

(ii) If $x$ belongs to the interior of $U_{f}$ for $f \in S L^{2}(G)$ and $x_{1}$ is an element of $G^{\#}$, then there exists some $u_{0}>0$ such that $0 \leqslant u \leqslant u_{0}$ implies $x \pm u x_{1}$ belongs to the interior of $U_{f}$.

Definition 4. If $f \in S L^{2}(G)$ then the function $f^{\square}$ defined on $U_{f} \times G^{*}$ by (*) will be called the $L^{2}$ Laplace transform of $f$. (The definition is to be interpreted in the $L^{2}$ sense and $f^{\square}$ can be considered as the limit as $n \rightarrow \infty$ of $f_{n}^{\square}(x, y)$ with $f_{n} \in C_{c}(G)$ and $f_{n} \rightarrow f$ in $L^{2}(G)$.)

ExAMPLE 1. Let $G=Z$, the group of integers with the discrete topology. 
Then $Z^{\#}=R$ and each element $x \in Z^{\#}$ can be identified with a positive real $r$ by the expression $x=\log r$. The element $\theta \in Z^{*}=T$ can be identified with a real number $\theta, 0 \leqslant \theta<1$. For $f \in S L^{2}(Z)$, and by obvious abuse of notation we have

$$
f^{\square}(r, \theta)=\sum_{-\infty}^{\infty} f(n) r^{n} e^{2 \pi i n \theta} \text {. }
$$

Hence the $L^{2}$ Laplace transform yields a formal Laurent series. It is not hard to see that the Laurent series actually converges absolutely for $x \in \operatorname{int}\left(U_{f}\right)$ and thus defines an analytic function on the interior of the annulus determined by $U_{f} \times T$.

EXAMPLE 2. Let $G=R$, the reals with the usual topology. Then $R^{\#}=R$ and if $f \in S L^{2}(R)$ then $f \in L^{1}(R)$ and its $L^{2}$ Laplace transform is the usual improper integral which defines an analytic function on $U \times R$ where $U$ is an open subset of $U_{f}$.

Differentiation. Partial differentiation of functions is introduced using oneparameter subgroups. If $f$ is a function defined on $G^{*}$ and if $x \in G^{\#}$, the directional derivative $f_{x}$ of $f$ at $y$ with respect to $x$ may be defined as $\lim _{u \rightarrow 0}(f(y x[u])-f(y)) / u$ whenever this limit exists.

Similarly if $F$ is defined on $G^{\#} \times G^{*}$, we can speak of the directional derivative $F_{\left(x_{1}, y_{1}\right)}$ of $F$ at $(x, y)$ with respect to $\left(x_{1}, y_{1}\right) \in G^{\#} \times G^{\#}$ by defining

$$
F_{\left(x_{1}, y_{1}\right)}(x, y)=\lim _{u \rightarrow 0}\left(F\left(x+u x_{1}, y y_{1}[u]\right)-F(x, y)\right) / u
$$

when this limit exists. Functions which satisfy (1) for all pairs $\left(x_{1}, y_{1}\right)$ shall be called differentiable. Given a real number $r$ and scalar multiplication $r\left(x_{1}, y_{1}\right)=$ $\left(r x_{1}, r y_{1}\right)$, it is clear that $F_{r\left(x_{1}, y_{1}\right)}=r F_{\left(x_{1}, y_{1}\right)}$. In this sense a differentiable function is real homogeneous. Now $G^{\#} \times G^{\#}$ can be converted into a complex vector space by defining $(u+i v)\left(x_{1}, y_{1}\right)=\left(u x_{1}-v y_{1}, u y_{1}+v x_{1}\right)$. However, it is not always true that if the limit (1) exists that equality (2) holds.

$$
F_{(u+i v)\left(x_{1}, y_{1}\right)}=(u+i v) F_{\left(x_{1}, y_{1}\right)} .
$$

Functions which satisfy (2) will be called complex homogeneous.

Definition 5. A function $F$ on $G^{\#} \times G^{*}$ is analytic $I$ if it is differentiable at all $(x, y) \in G^{\#} \times G^{*}$, complex homogeneous and continuous, with continuous directional derivatives.

REMARK. If $G=R$, then $G^{\#}=G^{*}=R$ and if we identify $G^{\#} \times G^{*}$ with the complex plane $C$, then $F$ is analytic $I$ on $G^{\#} \times G^{*}$ iff $F$ is analytic in the classical sense (per Cauchy).

More generally if $G=R^{n}$, then $G^{\#}=G^{*}=R^{n}$ and if $x, y$ are a pair of vectors such that $x+y=z=(1+i, \ldots, 1+i)$ and $\pi_{j} x=i$ or 1 , but not both, for each projection $\pi_{j}$, then the complex homogeneity condition implies $(\partial / \partial x+i \partial / \partial y) F=0$. 
It is also worthwhile to note that when dealing with groups satisfying Lemma 2, a function $F$ which is analytic I on $G^{\#} \times G^{*}$ automatically has continuous directional derivatives in a "dense set of directions".

Although analytic I functions have an inherent attractiveness, being in some sense the simplest generalizations of analytic functions, the Paley-Wiener problem is an $L^{2}$ problem and the related notion of analytic II functions must be introduced. (In the Appendix to our paper, it is shown that in general there is no reasonable condition which can be placed on $f \in S L^{2}(G)$ to insure that $f^{\square}$ is analytic I.)

Definition 6. Let $U$ be a subset of $G^{\#}$. If $F$ is defined on $U \times G^{*}$, then $F \in L^{2}\left(U \times G^{*}\right)$ iff for every $x \in U, F(x, y) \in L^{2}\left(G^{*}\right)$.

Definition 7. A function $F \in L^{2}\left(U \times G^{*}\right)$ is continuous II iff

$$
\|F(x, y)\|_{2}=\left(\int_{G^{*}}\left|F\left(x, y y_{1}\right)\right|^{2} d \mu\left(y_{1}\right)\right)^{1 / 2}
$$

is jointly continuous, where $d \mu$ is the normalized Haar measure on $G^{*}$.

Definition 8. Let $U$ be an open set of $G^{\#}$. Then $F \in L^{2}\left(U \times G^{*}\right)$ is differentiable II at $(x, y) \in U \times G^{*}$ iff $\lim _{u \rightarrow 0} F\left(x+u x_{1}, y y_{1}[u]-F(x, y)\right) / u$ exists as an $L^{2}$ function (in the sense of Definition 6) for all $\left(x_{1}, y_{1}\right) \in G^{\#} \times G^{\#}$.

Definition 9. Let $U$ be an open set of $G^{\#}$. Then $F \in L^{2}\left(U \times G^{*}\right)$ is analytic II in $U \times G^{*}$ iff $F$ is differentiable II at each $(x, y) \in U \times G^{*}$, complex homogeneous, and continuous II, and the directional derivatives are continuous II.

Definition 10. For $T$ any measurable set of $G^{*}$, with $0<\mu(T)<\infty$ and $F \in L^{2}\left(U \times G^{*}\right)$ define

$$
F_{T}(x, y)=\int_{T} F\left(x, y y_{1}\right) d \mu\left(y_{1}\right) .
$$

Lemma 6. Let $U \subset G^{\#}$ be open and $F$ analytic II on $U \times G^{*}$, and $T$ as above. Then $F_{T}$ is jointly continuous.

LEMmA 7. If $F$ is analytic II on $U \times G^{*}$, then $\left(F_{\left(x_{1}, y_{1}\right)}\right)_{T}=\left(F_{T}\right)_{\left(x_{1}, y_{1}\right)}$.

Proof. By definition there exists a family $\left\{H\left(x_{1}, y_{1}, u, x\right)(y)\right\}$ of $L^{2}$ functions on $G^{*}$ such that

$$
\left(F\left(x+u x_{1}, y y_{1}[u]\right)-F(x, y)\right) / u=H\left(x_{1}, y_{1}, u, x\right)(y)+F_{\left(x_{1}, y_{1}\right)}(x, y)
$$

with the additional property that $\lim _{u \rightarrow 0}\left\|H\left(x_{1}, y_{1}, u, x\right)\right\|_{2}=0$. Now $\left(F_{x_{1}, y_{1}}\right)_{T}$ $=\left(F_{T}\right)_{\left(x_{1}, y_{1}\right)}$ is equivalent to

$$
\begin{aligned}
\int_{T} F_{\left(x_{1}, y_{1}\right)}\left(x_{0}, y_{0} y\right) d \mu(y) & \\
& =\lim _{u \rightarrow 0} \int_{T}\left[H\left(x_{1}, y_{1}, u, x_{0}\right)\left(y_{0}\right)+F_{\left(x_{1}, y_{1}\right)}\left(x_{0}, y_{0} y\right)\right] d \mu(y)
\end{aligned}
$$

for all pairs $\left(x_{0}, y_{0}\right)$. This is immediate. 
Corollary 7.1. If $F$ is analytic II on $U \times G^{*}$ and $0<\mu(T)<\infty$ then $F_{T}$ is analytic I on $U \times G^{*}$.

For technical reasons, relating to the inverse Fourier transform, we make the following definition and prove an additional lemma.

Definition 11. For $x \in G^{\#}, y \in G^{*}$ and $t$ a fixed element of $G$, define

$$
E^{t}(x, y)=\exp (-x(t))\left\langle y^{-1}, t\right\rangle
$$

LEMMA 8. If $F$ is analytic II on $U \times G^{*}$ and $\sup _{x \in U}\|F(x, y)\|_{2}<M$ for some $M$, then $F E^{t}$ is analytic II on $U \times G^{*}$ for all $t \in G$, and

$$
\left(F E^{t}\right)_{\left(x_{1}, y_{1}\right)}=F_{\left(x_{1}, y_{1}\right)} E^{t}+F E_{\left(x_{1}, y_{1}\right)}^{t}
$$

(equality to be interpreted in the $L^{2}$ sense of Definition 7).

Proof. That $F E^{t} \in L^{2}\left(U \times G^{*}\right)$ is a trivial result. Analyticity II is proven in several steps. First the given equality is checked. The triangle inequality yields:

$$
\begin{gathered}
\left\|\left(F E^{t}\right)_{\left(x_{1}, y_{1}\right)}(x, y)-\left[F_{\left(x_{1}, y_{1}\right)} E^{t}(x, y)+F E_{\left(x_{1}, y_{1}\right)}^{t}(x, y)\right]\right\|_{2} \\
\leqslant \lim _{u \rightarrow 0} \|\left(\left[F\left(x+u x_{1}, y y_{1}[u]\right)-F(x, y)\right] / u\right) \\
\quad \times E^{t}\left(x+u x_{1}, y y_{1}[u]\right)-F_{\left(x_{1}, y_{1}\right)} E^{t}(x, y) \|_{2} \\
+\lim _{u \rightarrow 0} \|\left(F(x, y)\left[E^{t}\left(x+u x_{1}, y y_{1}[u]\right)-E^{t}(x, y)\right] / u\right) \\
-F E_{\left(x_{1}, y_{1}\right)}^{t}(x, y) \|_{2} .
\end{gathered}
$$

Using the error function $H\left(x_{1}, y_{1}, u, x\right)(y)$, the definition of $E^{t}$, the equality

$$
E_{\left(x_{1}, y_{1}\right)}^{t}(x, y)=E^{t}(x, y)\left(-\left(x_{1}(t)+i y_{1}(t)\right)\right),
$$

and the triangle equality, we see that for the sum (3),

$$
\begin{aligned}
(3) \leqslant & \lim _{u \rightarrow 0}\left\|F_{\left(x_{1}, y_{1}\right)} E^{t}(x, y)\left(\exp \left[-u\left(x_{1}(t)+i y_{1}(t)\right)\right]-1\right)\right\|_{2} \\
& +\lim _{u \rightarrow 0}\left\|E^{t}(x, y) \exp \left[-u\left(x_{1}(t)+i y_{1}(t)\right)\right] H\left(x_{1}, y_{1}, u, x\right)(y)\right\|_{2} \\
& +\lim _{u \rightarrow 0} \| F E^{t}(x, y)\left[\left(\exp \left[-u\left(x_{1}(t)+i y_{1}(t)\right)\right]-1\right) / u\right. \\
& \left.+\left(x_{1}(t)+i y_{1}(t)\right)\right] \|_{2} .
\end{aligned}
$$

Now the properties of the error function $H$ along with dominated convergence, allow us to conclude that this last sum converges to zero, and this proves the stated equality. 
Complex homogeneity follows easily from the functional equality in the statement of the lemma. It only remains to verify continuity II of $F E^{t}$ and the directional derivatives. Continuity of $F E^{t}$ follows from the triangle inequality and the choice of neighborhoods $N_{1}, N_{2}$ and $N_{3}$ at a point $\left(x_{0}, y_{0}\right) \in U \times G^{*}$, defined as follows. Let

$$
N_{1}=\left\{(x, y) \in U \times G^{*}:\left|\exp (-x(t))-\exp \left(-x_{0}(t)\right)\right|<\epsilon / 3 M\right\}
$$

(for the $t \in G$ used in the definition of $E^{t}$ and the $M$ of Lemma 8). Let

$$
N_{2}=\left\{(x, y) \in U \times G^{*}:\left|\left\langle y^{-1}, t\right\rangle-\left\langle y_{0}^{-1}, t\right\rangle\right|<\epsilon \exp \left(-x_{0}(t)\right) / 3 M\right\} .
$$

Let

$$
N_{3}=\left\{(x, y) \in U \times G^{*}:\left\|F(x, y)-F\left(x_{0}, y_{0}\right)\right\|_{2}<\epsilon \exp \left(-x_{0}(t)\right) / 3 .\right.
$$

Then for $(x, y) \in N_{1} \cap N_{2} \cap N_{3},\left\|F E^{t}(x, y)-F E^{t}\left(x_{0}, y_{0}\right)\right\|_{2}<\epsilon$. Continuity II of the directional derivatives follows similarly.

Corollary 9.1. If $F$ is analytic II on $U \times G^{*}$, then $\left(F E^{\dagger}\right)_{T}$ is analytic I on $K \times G^{*}$, where $K$ is a compact subset of $U$.

Study of $D$. To be able to estimate the magnitude of certain integrals, it is important to know that neither $G$ nor $G^{\#}$ is too large. Specifically, if $G$ is generated by the support of a strongly $L^{2}$. function, then $G^{\#}$ is finite dimensional (as a real vector space). This in turn imposes conditions on the size of $G$. Since $R^{n}$ is already finite dimensional, in this section we restrict our attention to $D$ and $D^{\#}$ (notation of Lemma 2).

Definition 12. For $t \in G$ define $[t]=\left\{s \in G: x(s)=a_{s t} x(t) \cdot \forall x \in G^{\#}\right\}$, where $a_{\text {st }}$ depends only on $s$ and $t$.

LEMma 9. For each $t \in G,[t]$ is a subgroup of $G$.

LEMMA 10. Let $D$ be a torsion-free discrete group. Then there exists a family $S$ of elements of $D$, such that $D$ can be expressed as the weak direct product of the $\left[t_{\lambda}\right], t_{\lambda} \in S$ (notation due to Hewitt and Ross [4]). We write this as $D \approx \Pi_{S}^{*}\left[t_{\lambda}\right]$.

Proof. The lemma follows by an immediate application of Zorn's lemma.

LEMMA 11. Let $S$ be as above. Given any set $\left\{a_{\lambda}\right\}$ of real numbers with $\lambda \in|S|$, there exists a linear functional $x \in D^{\#}$ such that $x\left(t_{\lambda}\right)=a_{\lambda}$.

Proof. For each $t_{\lambda} \in S$ define $x_{\lambda} \in D^{\#}$ by $x_{\lambda}\left(t_{\lambda}\right)=a_{\lambda}, x_{\lambda}\left(t_{\tau}\right)=0$, $\tau \neq \lambda$. The linear extension determines a unique linear functional $x_{\lambda}$. Set 
$x(t)=\Sigma_{S} x_{\lambda}(t)$. The sum is well defined since it is actually finite for every $t \in$ $D$, and $x$ is the required functional.

Definition 13. Let $G$ be a locally compact Abelian group and $f$ a function on $G$. Define $G_{f}$ to be the subgroup of $G$ generated by the support of $f$.

Definition 14. Let $G$ be a locally compact Abelian group. $G$ is finite dimensional iff $G^{\#}$ is a finite dimensional vector space.

LEMMA 12. Let $D$ be a discrete torsion-free Abelian group, and $f \in S L^{2}(D)$. Then $D_{f}$ is finite dimensional.

Proof. To prove this lemma, $S$ is chosen as in Lemma 10, and $\phi$ is chosen to be the isomorphism of $D$ onto its weak direct product representation $\Pi_{S}^{*}\left[t_{\lambda}\right]$. Let $\pi_{\lambda}$ be the projection from $\Pi_{S}^{*}\left[t_{\lambda}\right]$ onto $\left[t_{\lambda}\right]$. It then follows that $\left.\pi_{\lambda} \phi\right|_{D_{f}}(t)$ is identically zero except for finitely many $\lambda$, and hence a set $\left\{t_{1}, \ldots, t_{n}\right\} \subset S$ can be chosen such that $D_{f}$ can be isomorphically imbedded in $\left[t_{1}\right] \oplus \cdots \oplus\left[t_{n}\right]$. If this were not so, then $D_{f}$ could not be imbedded in a finite direct product as above. We could then form an infinite chain of sets, $N_{i}(i=1, \ldots)$ of elements of $D, N_{i}=\left\{t_{1}, \ldots, t_{i}\right\}, t_{i} \in S$, such that for every $t_{i}$ there exists $t(i) \in\left[t_{i}\right]$ with $f(t(i))=a_{i} \neq 0$ and furthermore $D_{f}$ cannot be embedded in $\Pi_{N_{i}}^{*}\left[t_{\lambda}\right]$ for any finite $i$. Since $D^{\#}$ has the compact-open topology, there exists an integer $n$ such that $x\left(N_{n}\right)=0$ implies that $x \in U_{f}$ (Lemma 5). We can define $x \in D^{\#}$ by $x\left(t_{i}\right)=0$ for $i=1, \ldots, n ; x(t(n+i))=1 / a_{i}$; and $x\left(t_{\lambda}\right)=0$ for $\lambda \notin \bigcup_{i} N_{i}$. Extending linearly, we define $x$ uniquely, and it is immediate that $x \in U_{f}$. However,

$$
\begin{aligned}
\sum_{D}|f(t) \exp x(t)|^{2} & \geqslant \sum_{D_{f}}|f(t) \exp x(t)|^{2} \\
& \geqslant \lim _{n \rightarrow \infty} \sum_{i=1, \ldots, n}|f(t(i)) x(t(i))|^{2}=\infty
\end{aligned}
$$

yielding a contradiction.

COROLlaRY 12.1. $D^{\#} I_{D_{f}}$ and $D_{f}^{\#}$ are both finite dimensional, and moreover, $\left.D^{\#}\right|_{D_{f}}=D_{f}^{\#}$.

Direct theorem. We shall now prove that the $L^{2}$ Laplace transform of a function $f \in S L^{2}(G)$ is an analytic II function. Our main tool is the dominated convergence theorem in conjunction with our earlier remarks that the support of $f$ is finite dimensional.

Definition 15. A function $F$ on $U \times G^{*}$ is uniformly $L^{2}$ on $U$ if $\sup _{x \in U}\|F(x, y)\|_{2}$ is bounded.

THEOREM 1. If $G$ is a locally compact Abelian group and if $f \in S L^{2}(G)$ then the $L^{2}$ Laplace transform 


$$
f^{\square}(x, y)=\int_{G} f(t) \exp (x(t))\langle y, t\rangle d \mu(t)
$$

(where $d \mu$ is the Haar measure on $G$ ), is analytic II on the interior of $U_{f} \times G^{*}$ and is uniformly $L^{2}$ on any compact subset $K$ of $U_{f}$. Moreover

$$
f_{\left(x_{1}, y_{1}\right)}^{\square}(x, y)=\int_{G} f(t) \exp x(t)\left(x_{1}(t)+i y_{1}(t)\right)\langle y, t\rangle d \mu(t) .
$$

In both the above formulas the equality is understood in the $L^{2}$ sense.

Proof. Formally we write

$$
F_{\left(x_{1}, y_{1}\right)}(x, y)=\int_{G} f(t) \exp x(t)\left(x_{1}(t)+i y_{1}(t)\right)\langle y, t\rangle d \mu(t),
$$

and verify that $F_{\left(x_{1}, y_{1}\right)}(x, y)$ is square integrable (with respect to $y \in G^{*}$ ) for any fixed $x$ in the interior of $U_{f}$. By Plancherel's theorem it suffices to show $x_{1} f \exp x$ and $y_{1} f \exp x$ are square integrable over $G$. Set $G_{1}=\left\{t \in G: x_{1}(t)\right.$ $\geqslant 0\}$ and $G_{2}=G \sim G_{1}$.

Choose $u>0$ sufficiently small so that $x \pm u x_{1}$ belongs to $\operatorname{int}\left(U_{f}\right)$ (the interior of $\left.U_{f}\right)$. On $G_{1},\left|(f \exp x) u x_{1}\right| \leqslant\left|f \exp \left(x+u x_{1}\right)\right|$ and on $G_{2}$, $\left|(f \exp x) u x_{1}\right| \leqslant\left|f \exp \left(x-u x_{1}\right)\right|$. The square integrability of the right-hand terms proves that $x_{1} f \exp x \in L^{2}(G)$. A similar argument shows that $y_{1} f \exp x$ $\in L^{2}(G)$.

Next we show that the difference quotient

$$
\left.f^{\square}\left(x+u x_{1}, y y_{1}[u]\right)-f^{\square}(x, y)\right) / u
$$

tends to $F_{\left(x_{1}, y_{1}\right)}(x, y)$ in the $L^{2}$ norm. Again by Plancherel's theorem we fix $x \in \operatorname{int}\left(U_{f}\right)$ and look at

$$
\lim _{u \rightarrow 0}\left(\int_{G}\left|(f \exp x)\left[\left(x_{1}+i y_{1}\right)-\left(\exp \left(u x_{1}+i u y_{1}\right)-1\right) / u\right]\right|^{2} d \mu(t)\right)^{1 / 2} .
$$

By use of the mean value theorem our formula becomes

$$
\lim _{u \rightarrow 0}\left(\int_{G}\left|(f \exp x)\left(x_{1}+i y_{1}\right)\left(1-\exp u^{*}(t)\left(x_{1}+i y_{1}\right)\right)\right|^{2} d \mu(t)\right)^{1 / 2}
$$

where for every $t \in G, 0<u^{*}(t)<u$. Dominated convergence yields the required results. Complex homogeneity follows from equation (4).

We now turn to showing that $f^{\square}(x, y)$ is continuous II. Choose $x_{0}$ in int $U_{f}$. Then by Plancherel's theorem we see

$$
\begin{aligned}
&\left\|f^{\square}\left(x_{0}, y_{0}\right)-f^{\square}(x, y)\right\|_{2}=\left\|f\left(\exp x_{0}-\exp x\left\langle y_{0}^{-1} y, t\right)\right)\right\|_{2} \\
& \leqslant\left\|\left(1-\left\langle y_{0}^{-1} y, t\right\rangle\right) f \exp x_{0}\right\|_{2}+\left\|f\left(\exp x_{0}-\exp x\right)\right\|_{2} .
\end{aligned}
$$

Since $G^{*}$ has the compact-open topology and the measure on $G$ is regular, and $\left|1-\left\langle y_{0}^{-1} y, t\right\rangle\right| \leqslant 2$, we see that there exists compact $K \subset G$ such that both the expressions 
and

$$
\int_{K}\left|\left(1-\left\langle y_{0}^{-1} y, t\right)\right) f(t) \exp x_{0}(t)\right|^{2} d \mu(t)
$$

$$
\int_{G \sim K}\left|\left(1-\left\langle y_{0}^{-1}, t\right\rangle\right) f(t) \exp x_{0}(t)\right|^{2} d \mu(t)
$$

are arbitrarily small for $y$ sufficiently close to $y_{0}$. Thus the first summand in (5) tends to zero.

To show that the second summand becomes small as $x$ converges to $x_{0}$, we use the equality $[6, \mathrm{pp} .130-132]$

$$
\begin{aligned}
\int_{G}|f(t) \exp x(t)|^{2} d \mu(t) & =\int_{G / N} \int_{N}|f(r s) \exp x(r s)|^{2} d \mu(r) d \mu(s) \\
& =\int_{R^{n} \oplus D} \int_{N}|f(r s) \exp x(r s)|^{2} d \mu(r) d \mu(s) \\
& =\int_{R^{n} \oplus D_{f}} \int_{N}|f(r s) \exp x(r s)|^{2} d \mu(r) d \mu(s)
\end{aligned}
$$

where $d \mu(r)$ and $d \mu(s)$ are the Haar measures on the groups $N$ and $G / N$ respectively. We can imbed the group $R^{n} \oplus D_{f}$ into the group $\left[t_{1}\right] \oplus \cdots \oplus\left[t_{n}\right] \oplus\left[t_{n+1}\right]$ $\oplus \cdots \oplus\left[t_{n+m}\right]$ where each $\left[t_{i}\right], i=1, \ldots, n$, is naturally isomorphic to the copies of $R$ in $R^{n}$, under the identification $t_{i} \sim e_{i}$, where $\left\{e_{i}\right\}$ is the natural basis for $R^{n}$, and $\left[t_{n+1}\right] \oplus \cdots \oplus\left[t_{n+m}\right]$ is the decomposition associated to $D_{f}$ as per Lemma 10. Define $\left[t_{i}\right]^{+}=\left\{t \in\left[t_{i}\right]: a_{t t_{i}} \geqslant 0\right\}$ and $\left[t_{i}\right]^{-}=\left\{t \in\left[t_{i}\right]: a_{t t_{i}}\right.$ $\leqslant 0\}$. Let $\gamma$ be a function from $\{1, \ldots, n+m\}$ to $\{+,-\}$. For any such $\gamma$, there exists $x_{\gamma} \in G^{\#}$ with $x_{\gamma}(t) \geqslant 0$ for $t \in Q_{\gamma}$, where $Q_{\gamma}$ is the quadrant $\Pi_{i=1}^{n+m}\left[t_{i}\right]^{\gamma(i)}, x_{\gamma}\left(t_{i}\right) \neq 0$ for $i=1, \ldots, n+m$ and $\left(x_{0}+x_{\gamma}\right) \in \operatorname{int}\left(U_{f}\right)$. Hence by dominated convergence, there exists $\delta_{\gamma}>0$ such that on $Q_{\gamma}$,

$$
\left\|f \exp x_{0}\left(1-\exp \delta_{\gamma} x_{\gamma}\right)\right\|_{2}<\epsilon / 2^{n+m} \text {. }
$$

(The $L^{2}$ norm is taken only over $Q_{\gamma}$.) Set $\delta=\min _{\gamma, i}\left|\delta_{\gamma} x_{\gamma}\left(t_{i}\right)\right|$. For $x \in N_{x_{0}}=$ $\left\{x \in G^{\#}:\left|x\left(t_{i}\right)\right|<\delta, i=0, \ldots, n+m\right\}$ we can conclude $\| f\left(\exp x_{0}-\right.$ $\left.\exp \left(x_{0}+x\right)\right) \|_{2}<\epsilon$. This proves continuity II. The fact that $\|F(x, y)\|_{2}$ is uniformly bounded on $K \times G^{*}$, where $K$ is a compact subset of $\operatorname{int}\left(U_{f}\right)$, follows immediately.

Continuity II of the directional derivatives is proved similarly.

Converse for $R^{n}$. Theorem 1 contains the statement of one direction of the Paley-Wiener theorem for locally compact Abelian groups. The converse statement will be proved in the following sections by first proving the results for special groups and then "glueing" the results together.

The first important locally compact group that will be considered is $R^{n}$. For this group, numerous proofs of the Paley-Wiener theorem have appeared: see 
Yoshida [16], Stein and Weiss [14], or Donoghue [1]. However, the proof that is most easily reformulated to include the concept of analyticity II is the proof that appears in Rudin [12, p. 368]. The full details of the reformulation will not be given here. The theorem will be stated and a few arguments indicated.

THEOREM 2. Let $G=R^{n}, U$ be an open set of $G^{\#}=R^{n}$ and $F$ be analytic II on $U \times G^{*}=U \times R^{n}$. Then there exists an $f \in S L^{2}\left(R^{n}\right)$ with $U_{f} \supset U$ such that $f^{\square}(x, y)=F(x, y)$ in the $L^{2}$ sense.

Proof. Heuristically, we write $f(t)=\int_{R^{n}} F(x, y) E^{t}(x, y) d \mu(y)$ with $d \mu$ the Lebesgue measure on $R^{n}$, and observe that if $f$ is well defined (as an $L^{2}$ function) then $f \in L^{2}\left(R^{n}\right)$ and $f \exp x \in L^{2}\left(R^{n}\right)$. Thus the crux of the proof is to show that the definition of $f$ is independent of $x \in U$. If $x_{1}$ and $x_{2}$ are any two points of $U$, then there exists a compact set $K \subset U$ with $0, x_{1}, x_{2}$ all belonging to $K$. Thus it suffices to show that $f$ defined with respect to $x \in K$ is equal to $f$ defined with respect to $0 \in K$. The major departure from Rudin's proof is the two-fold use of a diagonal argument.

Analysis of $F(x, y)$ on $D$. It was shown previously that if $f \in S L^{2}(D)$, where $D$ is a discrete torsion-free Abelian group, then $D_{f}$ is a finite dimensional group, and $f^{\square}$ is actually a function of a coset space. More specifically, if $D_{f}$ is imbedded in $\left[t_{1}\right] \oplus \cdots \oplus\left[t_{n}\right]$, then $f^{\square}$ is a function of the coset space $D^{\#} / N\left(t_{1}\right) \cap \cdots \cap N\left(t_{n}\right) \times D^{*} / I\left(t_{1}\right) \cap \cdots \cap I\left(t_{n}\right)$ where $N\left(t_{i}\right)$ is defined to be the set $\left\{x \in D^{\#}: x\left[t_{i}\right]=0\right\}$ and $I\left(t_{i}\right)$ is the set $\left\{y \in D^{*}: y\left[t_{i}\right]=1\right\}$. That $f^{\square}$ is a function on cosets can be expressed in still another way, which will depend on the following facts:

$$
\begin{aligned}
& \text { (i) } D \approx \Pi_{S}^{*}\left[t_{\lambda}\right] \text {. } \\
& \text { (ii) } x \in D^{\#} \text { can be written as } \Sigma_{|s|} x_{\lambda} \text { where }\left.x_{\lambda}\right|_{\left[t_{\lambda}\right]}=\left.x\right|_{\left[t_{\lambda}\right]} \text { and }\left.x\right|_{\left[t_{\tau}\right]} \\
& =0 \text {. (iii) } y \in D^{*} \text { can be written as } \Pi_{|s|} y_{\lambda} \text { where }\left.y_{\lambda}\right|_{\left[t_{\lambda}\right]}=\left.y\right|_{\left[t_{\lambda}\right]} \text { and }\left.y_{\lambda}\right|_{\left[t_{\tau}\right]} \\
& =1 \text {. }
\end{aligned}
$$

(iv) If $F$ is a function on $D^{\#} \times D^{*}$, then $F(x, y)$ can be written as $F\left(x_{1}, \ldots ; y_{1}, \ldots\right)$ (where both $x=\left(x_{1}, \ldots\right)$ and $y=\left(y_{1}, \ldots\right)$ may have uncountably many coordinates).

REMARK. The natures of the functional $x_{\lambda}$ and the character $y_{\lambda}$ are different insofar as $x_{\lambda}$ is uniquely determined by any nonzero value $x_{\lambda}(t)$ for some $t \in\left[t_{\lambda}\right]$ whereas $y_{\lambda}$ is not.

It is clear that in terms of coordinate notation, there exists an $n$ for which

$$
\begin{aligned}
f^{\square}\left(x_{1}, \ldots, x_{n}, 0, \ldots ; y_{1}, \ldots, y_{n}, 0 ; \ldots\right) \\
\quad=f^{\square}\left(x_{1}, \ldots, x_{n}, x_{n+1}, \ldots ; y_{1}, \ldots, y_{n}, y_{n+1}, \ldots\right) .
\end{aligned}
$$


The aim of this section is to prove this result for $F$ analytic II on $U \times D^{*} C$ $D^{\#} \times D^{*}$. Recalling that the images of the one-parameter subgroups of $D^{*}$ are dense in $D^{*}$ under the mapping which we will call $\psi$, we deal first with the space $D^{\#} \times \psi\left(D^{\#}\right)$.

Lemma 13. $A$ basis for $D^{\#}$ can be chosen such that $x \in D^{\#}$ and $y \in \psi\left(D^{\#}\right)$ can be written as $x=\left(r_{1}, r_{2}, \ldots\right)$ and $y=\left(r_{1}^{\prime}, r_{2}^{\prime}, \ldots\right)$, respectively, where $r_{i}$ and $r_{i}^{\prime}$ are all real numbers.

Proof. For any $t \in D, t \neq e,[t]^{\#}$ is one dimensional and hence can be identified with the reals $R$. Any $x \in D^{\#}$ can be expressed in terms of the functions $x_{\lambda}$ dual to the $t_{\lambda} \in S$.

It is not hard to see that on the hyperplane $R_{j} \times R_{j}^{\prime}$ determined by $r_{i}=0$, $r_{i}^{\prime}=0$ for $i \neq j$, the topology induced by the compact-open topology is locally the usual Euclidean topology. Thus if $F$ is analytic II on $D^{\#} \times D^{*}, F_{T}$ is analytic I and the restriction of $F_{T}$ to $R_{j} \times R_{j}^{\prime}$ denoted $F_{\left.T\right|_{R_{j}} \times R_{j}^{\prime}}$ is analytic in the classical sense.

LEMma 14. Let $D$ be as above, $U$ a convex neighborhood of $0 \in G^{\#}$, and let $F$ be analytic II on $U \times D^{*}$. Let $T$ be a measurable subset of $D^{*}$ with $0<$ $\mu(T)<\infty$ and $F_{T}$ as in Definition 10. Then in terms of the above remarks, for each point $(x, y) \in U \times \psi\left(D^{\#}\right)$, there exists a neighborhood $N_{(x, y)}$ of $(x, y)$ and an integer $n=n(x, y)$ for which

$$
\begin{aligned}
F_{T}\left(r_{1}, \ldots, r_{n}, 0, \ldots ; r_{1}^{\prime}, \ldots, r_{n}^{\prime}, 0, \ldots\right) & \\
& =F_{T}\left(r_{1}, \ldots, r_{n}, r_{n+1}, \ldots ; r_{1}^{\prime}, \ldots, r_{n}^{\prime}, r_{n+1}^{\prime}, \ldots\right)
\end{aligned}
$$

as functions on $N_{(x, y)} \cap \psi\left(D^{\#}\right)$.

Proof. Without loss of generality the lemma is proved at $(x, y)=(0,0)$. Since both $D^{\#}$ and $\psi\left(D^{\#}\right)$ are topologized with the compact-open topology, there exists an integer $n$ and real numbers $M$ and $\epsilon>0$ such that if $\left|r_{i}\right|<\epsilon$ and $\left|r_{i}^{\prime}\right|<\epsilon$ for $i=1, \ldots, n$, then $x=\left(r_{1}, \ldots, r_{n}, r_{n+1}, \ldots\right)$ belongs to $U$ and by continuity of $F_{T}$,

$$
\begin{aligned}
\mid F_{T}\left(r_{1}, \ldots, r_{n}, r_{n+1}, \ldots ; r_{1}^{\prime}, \ldots,\right. & \left.r_{n}^{\prime}, r_{n+1}^{\prime}, \ldots\right) \\
& -F_{T}(0, \ldots ; 0, \ldots) \mid<M .
\end{aligned}
$$

In particular $r_{n+j}$ and $r_{n+j}^{\prime}$ can be allowed to range over all of $R$. Now consider the multiply indexed family of analytic functions

$$
\begin{aligned}
F_{n+j}^{\left(\mathbf{r}, \mathbf{r}^{\prime}\right)}\left(r_{n+j}, r_{n+j}^{\prime}\right)= & F_{T}\left(r_{1}, \ldots, r_{n+j}, 0, \ldots ; r_{1}^{\prime}, \ldots, r_{n+j}^{\prime}, 0, \ldots\right) \\
& -F_{T}(0, \ldots ; 0, \ldots),
\end{aligned}
$$


where $j \geqslant 0$ and $\left|r_{i}\right|<\epsilon$ and $\left|r_{i}^{\prime}\right|<\epsilon$ for $i=1, \ldots, n$. By Liouville's theorem each $F_{n+j}^{\left(\mathbf{r}, \mathbf{r}^{\prime}\right)}$ is a constant. By virtue of the continuity of $F_{T}$ and the appropriate telescoping series, the lemma follows.

COROLlARY 14.1. The integer $n=n(x, y)$ is independent of the point $(x, y)$.

Proof. Let $n=n(0,0)$. Then by analytic continuation

$$
F_{T}\left(x_{1}, \ldots ; x_{1}^{\prime}, \ldots\right)-F_{T}\left(x_{1}, \ldots, x_{n}, 0, \ldots ; x_{1}^{\prime}, \ldots, x_{n}^{\prime}, 0, \ldots\right)
$$

is identically zero $[3, \mathrm{p} .6]$.

COROLlary 14.2. The function $F_{T}$ can be regarded as a function on the

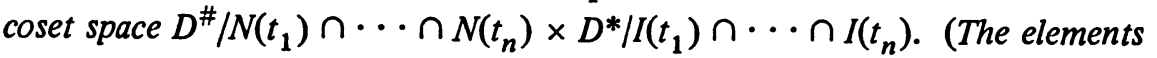
$t_{i}$ are those elements of $D$ used in the direct sum representation (i) which furthermore determine the functionals $x_{i}$ used to calculate $r_{i}$ and $r_{i}^{\prime}, i=1, \ldots, n$, in Lemma 14.)

Proof. The corollary follows from the denseness of $\psi\left(D^{\#}\right)$ in $D^{*}$ and from Corollary 14.1.

Converse for $D$. Let $t_{1}, \ldots, t_{n}$ be elements of $D$ as in Corollary 14.2. Let $D_{F}^{\#}=\left[t_{1}\right]^{\#} \times \cdots \times\left[t_{n}\right]^{\#}$, where $\left[t_{i}\right]^{\#}=\left\{x \in D^{\#}: x\left(t_{j}\right)=0, j \neq i\right\}$. Define a function $b: D_{F}^{\#} \times D^{\#} \rightarrow R$ by $b(x, y)=\sum_{i=1}^{n} r_{i} s_{i}$ where $x=\left(r_{1}, \ldots\right.$, $\left.r_{n}, 0, \ldots\right)$ and $y=\left(s_{1}, \ldots, s_{n}, s_{n+1}, \ldots\right)$ as in Lemma 14. The function $b$ determines a projection of $D^{\#}$ onto $D_{F}^{\#}$. Now if $K$ is a compact subset of $D_{F}^{\#}$ and if $F$ is analytic II on $U \times D^{*}$, then there exists an integer $M_{k}$ for which $\|F(x, y)\|_{2} \leqslant M_{k}$ for all $x \in U$ whose projection on the subspace $D_{F}^{\#}$ lies in $K$. Conversely, if $U$ is a neighborhood of 0 in $D^{\#}$ and $x \in U$, there exists a compact neighborhood $K_{x}$ of zero in $D_{F}^{\#}$ such that the projection of $x$ on $D_{F}^{\#}$ lies in the interior of $K_{x}$. These facts prove the following

LEMMA 15. If $U$ is a convex neighborhood of $0 \in D^{\#}$ and $F$ is analytic II on $U \times D^{*}$, then for every $(x, y) \in U \times D^{*}$, there exists a neighborhood $U_{x}$ of $x$ and 0 such that $F E^{t}(x, y)$ is analytic II on $U_{x} \times D^{*}$.

We now state and prove the converse of Theorem 1 .

THEOREM 3. Let $D$ be a discrete torsion-free Abelian group, $U$ an open convex neighborhood of 0 in $D^{\#}$ and $F$ an analytic II function on $U \times D^{*}$. Then there exists a function $f \in S L^{2}(D)$ with $U \subset U_{f}$ and $f^{\square}(x, y)=F(x, y)$ (in the $L^{2}$ sense) for $x \in U$.

Proof. As in the case for $R^{n}$, heuristically we write the equality 
$f(t)=\int_{D^{*}} F E^{t}(x, y) d \mu(y)$ where $d \mu(y)$ is the Haar measure on $D^{*}$. Again the burden of the proof is to show that this definition is independent of $x \in U$. It suffices to show $\int_{D^{*}} F E^{t}(0, y) d \mu(y)=\int_{D^{*}} F E^{t}(x, y) d \mu(y)$. Choose $U_{x}$ as in the previous remarks. Since $D$ is discrete, $D^{*}$ is compact $[4$, p. $362,(23.17)]$. By setting $T=D^{*}$, it follows that $\left(F E^{t}(x, y)\right)_{T}$ is analytic $I$ and due to the invariance of the Haar measure is independent of $y$ (for fixed $x \in U_{x}$ ). As a result of the homogeneity condition, the directional derivatives $\left(F E^{t}(x, y)\right)_{T_{\left(x_{1}, 0\right)}}$ are identically zero for all $x_{1}$ in $D^{\#}$, and thus because of the finite dimensional nature of $F E^{t}$ we have the equality $\int_{D^{*}} F E^{t}(0, y) d \mu(y)=\int_{D^{*}} F E^{t}(x, y) d \mu(y)$. This equality holds not only in the $L^{2}$ sense but also in the pointwise sense for either of the following two reasons: (i) $F \in L^{2}\left(D^{*}\right) \rightarrow F \in L^{1}\left(D^{*}\right)$ or (ii) each point of $D$ has unit mass.

The other assertions of the theorem follow easily.

Converse for $N$. Since there exist no nontrivial linear functionals on $N$, the expression $\int_{N^{*}} F E^{t}(x, y) d \mu(y)$ is simply the inverse Fourier transform and is independent of $x$ as $x$ is identically zero (Corollary 2.1).

Glueing. In this section the results for $N, D$ and $R^{n}$ are "glued" together to prove the converse direction for a locally compact Abelian group $G$.

LEMMA 16. Let $G$ be a group satisfying the conditions of Lemma 2, and $G^{\#}$ and $G^{*}$ its associated space of linear functionals and character group respectively. Let $U$ be a convex open neighborhood of $0 \in G^{\#}$ and $F(x, y)$ an analytic II function on $U \times G^{*}$. Then there exists an $f \in S L^{2}(G)$ such that $U \subset U_{f}$ and $f^{\square}(x, y)=F(x, y)$ in the $L^{2}$ sense of Definition 7 .

Proof. Since there exists an integer $n$ such that $G \approx R^{n} \oplus D$, the integral $\int_{G^{*}} F E^{t}(x, y) d \mu(y)$ can be written $\int_{R^{n}} \int_{D^{*}} F E^{t}(x, y) d \mu_{D}\left(y_{D}\right) d \mu_{R}\left(y_{R}\right)$ where $d \mu_{D}\left(y_{D}\right)$ and $d \mu_{R}\left(y_{R}\right)$ are the Haar measures on $D^{*}$ and $R^{n}$, respectively, and the element $y \in G^{*}$ is identified with its direct sum expression $y=y_{D}+y_{R}$. (These integrals are to be interpreted in an $L^{2}$ sense and the steps are justified by $[6$, pp. 130-132] and $[4$, p. 362, (23.18)].)

Once again it must be shown that the function $f$ given by $f(t)=$ $\int_{G^{*}} F E^{t}(x, y) d \mu(y)$ is well defined. By Theorem 3, the integral $\int_{D} F E^{t}(x, y) d \mu_{D}\left(y_{D}\right)$ is independent of $\pi_{D}(x)$ where $\pi_{D}$ is the projection of $G^{\#}$ on $D^{\#}$. Now once it is established that this latter integral determines an analytic II function on $\pi_{R^{n}}(U) \times R^{n}$, the lemma follows from Theorem 2 .

However, analyticity II follows from the equality

$$
\lim _{u \rightarrow 0} \int_{R^{n}}\left|\int_{D^{*}} H\left(x_{1}, y_{1}, u, x\right)(y) d \mu_{D}\left(y_{D}\right)\right|^{2} d \mu_{R}\left(y_{R}\right)=0,
$$


where $x_{1} \in\left(R^{n}\right)^{\#}=R^{n}$ and $y_{1} \in\left(R^{n}\right)^{*}=R^{n}$, and $H$ is the error function of Lemma $7[15$, p. 271].

THEOREM 4. Let $N$ be as in Corollary 2.1 and let $G$ be a locally compact Abelian group and $G^{\#}, G^{*}$ its associated space of linear functionals and character group respectively. Let $U$ be a convex open neighborhood of $0 \in G^{\#}$ and $F(x, y)$ an analytic II function on $U \times G^{*}$. Then there exists an $f \in S L^{2}(G)$ such that $U \subset U_{f}$ and $f^{\square}(x, y)=F(x, y)$ in the $L^{2}$ sense of Definition 7.

Proof. Define $A\left(G^{*}, N\right)$ to be the set of elements of $G^{*}$ given by $\{y \in$ $\left.G^{*}: y(t)=1 \forall t \in N\right\}$. Then from [4, p. 380, (24.11)], $(G / N)^{*} \approx A\left(G^{*}, N\right)$ and from $\left[4\right.$, p. 365, (23.25)], $N^{*} \approx G^{*} \mid A\left(G^{*}, N\right)$. Hence by $[6$, pp. 130-132],

$$
\begin{aligned}
\int_{G^{*}} F E^{t}(x, y) d \mu(y) & =\int_{G^{*} / A\left(G^{*}, N\right)} \int_{A\left(G^{*}, N\right)} F E^{t}\left(x, y^{\prime}\right) d \mu\left(y^{\prime}\right) d \mu(\mathrm{y}) \\
& =\int_{N^{*}} \int_{(G / N)^{*}} F E^{t}\left(x, y^{\prime}\right) d \mu\left(y^{\prime}\right) d \mu(\mathrm{y}) .
\end{aligned}
$$

Theorem 4 follows immediately from this expression using Lemma 17 and the results for $G=N$.

We summarize our results below.

THEOREM 5. Let $G$ be a locally compact Abelian group. If $f \in S L^{2}(G)$, then its $L^{2}$ Laplace transform $f^{\square}$ is analytic II on the interior of $U_{f} \times G^{*}$ and

$$
f_{\left(x_{1}, y_{1}\right)}(x, y)=\int_{G} f(t) \exp x(t)\langle y, t\rangle\left(x_{1}(t)+i y_{1}(t)\right) d \mu(t)
$$

where the equality is an $L^{2}$ equality in the sense of Definition 7. Conversely, an analytic II function $F$ defined on $U \times G^{*}$ with $U$ a convex, open neighborhood of $0 \in G^{*}$, can be written as the $L^{2}$ Laplace transform of a function $f \in S L^{2}(G)$ with $U \subset U_{f}$

Appendix. It develops that we could have used a slightly different class of functions in formulating our theorem. A function $f \in L^{2}(G)$ is defined to be $M S L^{2}(G)$ (Mackey [7]) if the set $S \subset G^{\#}$ for which $f(t) \exp x(t) \in L^{2}(G)$ for $x \in S$ has the following two properties: (i) $S$ is convex and (ii) if $x \in S, \exists u>1$ with $u x \in S$. It is immediate that $f \in S L^{2}(G) \Rightarrow f \in M S L^{2}(G)$.

LEMmA A.1. Let $G$ be a locally compact Abelian group. Then the corresponding theorems of the text hold for $f \in M S L^{2}(G)$.

Proof. For $f \in M S L^{2}(G)$, let $U_{[f]}$ be the corresponding subset of $G^{\#}$ such that $f \exp x \in L^{2}(G)$ for $x \in U_{[f]}$. The main property of $U_{[f]}$ needed to prove the appropriate formulations of Theorems 1-5 of the text is that for all $x \in U_{[f]}$ and for every quadrant $Q_{i}$ of $D_{f} \oplus V$, there exists an $x_{i} \in U_{[f]}$ for 
which $x(t) \leqslant x_{i}(t)$ for every $t \in Q_{i}$. This property is obviously true.

The following lemmas are also easily verified.

LEMMA A.2. Let $G=R^{n}$ (or $Z^{n}$ ), $U$ be an open convex neighborhood of $0 \in G^{\#}$, and $F$ be an analytic II function on $U \times G^{*}$. Then there exists a function $f^{\square}$ which is analytic I on $U \times G^{*}$ and satisfies $\left\|F(x, y)-f^{\square}(x, y)\right\|_{2}=0$ for $x \in U$.

LEMmA A.3. Let $U$ be a convex open set of $R$. Let $F(x, y)$ be an analytic function defined on the strip of the complex plane determined by $U \times R$. If $\sup _{x \in U}\|F(x, y)\|_{2}=\sup _{x \in U}\left(\int_{R}|F(x, y)|^{2} d y\right)^{1 / 2}$ is bounded, then $F$ is analytic II.

Lemmas A.2 and A.3 assert the "essential equivalence" of the classical Paley-Wiener theorem and the analytic II version.

In view of the above lemmas one might be led to conclude that if $G$ is a locally compact Abelian group, and $F$ is an analytic II function on $U \times G^{*}$ (where $U$ is a convex open set of $G^{\#}$ ) that then there exists a function $\mathbf{F}$ analytic $I$ on $U \times G^{*}$ or some "slightly smaller domain" with $\|\mathrm{F}(x, y)-F(x, y)\|_{2}=0$. This conjecture is false, as the following example shows.

EXAmpLE A.1. Let $G=Q_{d}$, the group of additive rationals with the discrete topology. Consider the function $f: Q_{d} \rightarrow R$ defined by

$$
\begin{aligned}
f(1 / n) & =1 / n, \\
f(x) & =0 \quad \text { for all other } x .
\end{aligned}
$$

Clearly $f \in S L^{2}\left(Q_{d}\right)$ and in fact $U_{f}=(-\infty, \infty)$ and

$$
f^{\square}(x, y)=\int_{Q_{d}} f(t) e^{x t}\langle y, t\rangle d t=\sum_{Q_{d}} f(t) e^{x t}\langle y, t\rangle .
$$

If $\langle y, t\rangle$ is of the form $e^{i r t}$ for some $r \in R$, it is not hard to see that $f^{\square}(x, y)$ is unbounded for all $x \geqslant 0$. Now assume for the moment that $\mathrm{F}(x, y)$ is an analytic I function on $U \times Q_{d}^{*}$ that satisfies $\left\|\mathrm{F}(x, y)-f^{\square}(x, y)\right\|_{2}=0$ for each $x$ belonging to some compact neighborhood $K$ of 0 . Then there exists a function $\mathrm{f} \in$ $S L^{2}\left(Q_{d}\right)$ such that $\mathrm{f}^{\square}(x, y)=\mathrm{F}(x, y)$ ( $L^{2}$ equality). By Plancherel's theorem, we can conclude that $\|\mathrm{f}-f\|_{2}=0$. However, this implies $\mathbf{f}=f$ pointwise, as $Q_{d}$ carries the point measure.

REMARK. The above example shows that although $R$ is dense in $Q_{d}^{*}$, it has measure zero. ( $R$ is imbedded in $Q_{d}^{*}$ through the identification $r \leftrightarrow e^{i r(\cdot)}$, and $Q_{d}^{*}$ is the Bohr compactification of this imbedded copy of $R$.)

EXAMPle A.2. Let $Q_{d}$ and $f$ be as before. Then $f \in S L^{2}\left(Q_{d}\right)$, but $f \notin$ $L^{1}\left(Q_{d}\right)$.

One might conjecture now that if $f \in S L^{2} \cap L^{1}\left(Q_{d}\right)$ and $x \in \operatorname{int}\left(U_{f}\right)$, then 
$f \exp x \in L^{1}\left(Q_{d}\right)$. Intuitively speaking, the $S L^{2}$ property governs the global growth and the $L^{1}$ property governs the local growth, so the conjecture might seem plausible. Nevertheless, it proves false.

EXAMPLE A.3. We define $f$ in terms of its distribution and $L^{2}$ norm. We choose numbers $\eta(n)$ and $N(n)$ and construct a function $f \in L^{2}\left(Q_{d}\right)$ as follows. Let $I_{n}$ be the half-open interval $[n, n+1), \eta(n)=\Sigma_{I_{n}}|f(x) \exp (n+1)|^{2}$, and $N(n)=\mu\left(\right.$ support $\left.\left.f\right|_{I_{n}}\right)(\mu$ is the point measure).

Set

$$
\begin{aligned}
& \eta(n)=1 /(n \exp ((n+1) / 2))^{2} \text { for } n \geqslant 1, \\
& \eta(n)=0 \quad \text { otherwise. }
\end{aligned}
$$

Define also

$$
\begin{aligned}
& N(n)=[1 / \eta(n)] \text { for } n \geqslant 1, \\
& N(n)=0 \quad \text { otherwise. }
\end{aligned}
$$

(We use $[1 / \eta(n)]$ to signify the integral part of $1 / \eta(n)$.)

Choose a set $S(n)$ of $N(n)$ points in $I_{n}$ and define

$$
\begin{aligned}
& f(x)=\eta(n)^{1 / 2} / N(n)^{1 / 2} \exp (n+1) \text { for } x \in I_{n} \cap S(n), n=1,2, \ldots, \\
& f(x)=0 \text { otherwise. }
\end{aligned}
$$
from

We claim $U_{f} \supset(-\infty, 3 / 2]$. It suffices to show that $3 / 2 \in U_{f}$, which follows

$$
\begin{aligned}
\sum_{Q_{d}}|f(t) \exp s t|^{2} & =\sum_{n} \sum_{I_{n}}|f(t) \exp s t|^{2} \\
& \leqslant \sum_{n} \sum_{I_{n}}\left(\frac{\exp (n+1) s}{N(n) n \exp (3(n+1) / 2)}\right)^{2} \\
& =\sum_{n}\left(\frac{\exp (n+1) s}{n \exp (3(n+1) / 2)}\right)^{2} .
\end{aligned}
$$

This last sum clearly converges for $s=3 / 2$.

We can show additionally that $f \in L^{1}$ because

$$
\sum_{Q_{d}}|f(t)|=\sum_{n} \frac{(\eta(n) N(n))^{1 / 2}}{\exp (n+1)}<\infty
$$

(since $\eta(n) N(n)<2)$.

However, for $s \geqslant 1, f \exp s \notin L^{1}$. We show that $\Sigma_{Q_{d}}|f(t) \exp t|$ diverges:

$$
\begin{aligned}
\sum_{Q_{d}}|f(t) \exp t| & =\sum_{n} \sum_{I_{n}}|f(t) \exp t| \\
& \geqslant \sum_{n>1}(\eta(n) N(n))^{1 / 2} \frac{\exp n}{\exp (n+1)}=\frac{1}{e} \sum_{n>1}(\eta(n) N(n))^{1 / 2}
\end{aligned}
$$


and since $\eta(n) N(n)>1$, the sum diverges.

\section{BIBLIOGRAPHY}

1. W. F. Donoghue, Jr., Distributions and Fourier transforms, Academic Press, New York, 1969.

2. L. Fuchs, Infinite Abelian groups. Vol. 2, Academic Press, New York, 1973.

3. R. C. Gunning and H. Rossi, Analytic functions of several complex variables, Prentice-Hall, Englewood Cliffs, N.J., 1965. MR 31 \#4927.

4. E. Hewitt and K. A. Ross, Abstract harmonic analysis. Vol. I: Structure of topological groups. Integration theory, group representations, Die Grundlehren der math. Wissenschaften, Band 115, Academic Press, New York; Springer-Verlag, Berlin, 1963. MR 28 \# 158.

5. I. Kaplansky, Infinite Abelian groups, Univ. of Michigan Press, Ann Arbor, 1954. MR 16, 444.

6. L. H. Loomis, An introduction to abstract harmonic analysis, Van Nostrand, New York and London, 1953. MR 14, 883.

7. G. W. Mackey, The Laplace transform for locally compact Abelian groups, Proc. Nat. Acad. Sci. U.S.A. 34 (1948), 156-162. MR 9, 497.

8. D. Montgomery and L. Zippin, Topological transformation groups, 3rd printing, Interscience, New York, 1965.

9. R. E. A. C. Paley and N. Wiener, Fourier transforms in the complex domain, Amer. Math. Soc. Colloq. Publ., vol. 19, Amer. Math. Soc., Providence, R. I., 1934.

10. L. Pontrjagin, Topological groups, Princeton Math. Ser., vol. 2, Princeton Univ. Press, Princeton, N. J., 1939. MR 1, 44.

K. A. Ross, See E. Hewitt [4].

11. W. Rudin, Fourier analysis on groups, Interscience Tracts in Pure and Appl. Math., no. 12, Interscience, New York, 1962. MR 27 \#2808. \#1420.

12. - Real and complex analysis, McGraw-Hill, New York, 1966. MR 35

13. R. Saeks, Resolution space operators and systems, Springer-Verlag, Berlin, 1973.

14. E. M. Stein and G. Weiss, Introduction to Fourier analysis on Euclidean spaces, Princeton Math. Ser., no. 32, Princeton Univ. Press, Princeton, N. J., 1971. MR 46 \#4102.

15. E. M. Stein, Singular integrals and differentiability properties of functions, Princeton Math. Ser., no. 30, Princeton Univ. Press, Princeton, N. J., 1970. MR 44 \#280.

G. Weiss: See E. M. Stein [14].

16. K. Yoshida, Functional analysis, 2nd ed., Die Grundlehren der math. Wissenschaften, Band 123, Academic Press, New York; Springer-Verlag, Berlin, 1965. MR 31 \#5054.

DEPARTMENT OF MATHEMATICS, TEXAS TECH UNIVERSITY, LUBBOCK, TEXAS 79409 